\title{
The TetO rat as a new translational model for type 2 diabetic retinopathy by inducible insulin receptor knockdown
}

\author{
Nadine Reichhart ${ }^{1}$ - Sergio Crespo-Garcia ${ }^{1}$ - Nadine Haase ${ }^{2,3,4}$ - Michaela Golic ${ }^{2,4,5,6}$. \\ Sergej Skosyrski $^{1}$ • Anne Rübsam ${ }^{1,7}$ - Christina Herrspiegel ${ }^{1}$ - Norbert Kociok ${ }^{1}$. \\ Natalia Alenina $^{3,8} \cdot$ Michael Bader $^{3,4,8,9,10}$ - Ralf Dechend ${ }^{2,4,10,11}$ - Olaf Strauss ${ }^{1}$. \\ Antonia M. Joussen ${ }^{1}$
}

Received: 13 May 2016 / Accepted: 30 August 2016 /Published online: 4 October 2016

(C) Springer-Verlag Berlin Heidelberg 2016

\begin{abstract}
Aims/hypothesis Although the renin-angiotensin system plays an important role in the progression of diabetic retinopathy, its influence therein has not been systematically evaluated. Here we test the suitability of a new translational model of diabetic retinopathy, the TetO rat, for addressing the role of angiotensin-II receptor 1 (AT1) blockade in experimental diabetic retinopathy.

Methods Diabetes was induced by tetracycline-inducible small hairpin RNA (shRNA) knockdown of the insulin receptor in rats, generating TetO rats. Systemic treatment consisted of an AT1 blocker (ARB) at the onset of diabetes, following which, 4-5 weeks later the retina was analysed in vivo and ex vivo. Retinal function was assessed by Ganzfeld electroretinography (ERG).
\end{abstract}

Nadine Reichhart and Sergio Crespo-Garcia contributed equally to this study

Electronic supplementary material The online version of this article (doi:10.1007/s00125-016-4115-0) contains peer-reviewed but unedited supplementary material, which is available to authorised users.

Nadine Reichhart

nadine.reichhart@charite.de

$\triangle$ Antonia M. Joussen

antonia.joussen@charite.de

1 Department of Ophthalmology, Charité-Universitätsmedizin Berlin Campus Virchow Klinikum, Augustenburger Platz 1,

13353 Berlin, Germany

2 Experimental and Clinical Research Center, a cooperation between the Max Delbrück Center for Molecular Medicine in the Helmholtz Association and the Charité-Universitätsmedizin Berlin, Berlin, Germany

3 Max Delbrück Center for Molecular Medicine in the Helmholtz Association, Berlin, Germany

4 Berlin Institute of Health, Berlin, Germany
Results Retinal vessels in TetO rats showed differences in vessel calibre, together with gliosis. The total number and the proportion of activated mononuclear phagocytes was increased. TetO rats presented with loss of retinal ganglion cells (RGC) and ERG indicated photoreceptor malfunction. Both the inner and outer blood-retina barriers were affected. The ARB treated group showed reduced gliosis and an overall amelioration of retinal function, alongside RGC recovery, whilst no statistically significant differences in vascular and inflammatory features were detected.

Conclusions/interpretation The TetO rat represents a promising translational model for the early neurovascular changes associated with type 2 diabetic retinopathy. ARB treatment had an effect on the neuronal component of the retina but not on the vasculature.

5 Department of Obstetrics, Charité-Universitätsmedizin Berlin Campus Virchow Klinikum, Berlin, Germany

6 Department of Gynecology, Charité-Universitätsmedizin Berlin Campus Virchow Klinikum, Berlin, Germany

7 Department of Ophthalmology and Visual Sciences, University of Michigan, Ann Arbor, MI, USA

8 German Center for Cardiovascular Disease, Berlin, Germany

9 Department of Biology, Universität zu Lübeck, Lübeck, Germany

10 Charité-Universitätsmedizin Berlin Campus Berlin-Buch, Berlin, Germany

11 Department of Cardiology and Nephrology, HELIOS Klinikum Berlin-Buch, Berlin, Germany 
Keywords Angiotensin-II receptor 1 blockade $\cdot$ Experimental diabetic retinopathy $\cdot$ Neurodegeneration $\cdot$ Translational model $\cdot$ Type 2 diabetes

$\begin{array}{ll}\text { Abbreviations } & \\ \text { ACE } & \text { Angiotensin-converting enzyme } \\ \text { ARB } & \text { AT1 blocker } \\ \text { AT1 } & \text { Angiotensin-II receptor 1 } \\ \text { BRB } & \text { Blood-retina barrier } \\ \text { ERG } & \text { Electroretinography } \\ \text { GCL } & \text { Ganglion cell layer } \\ \text { GFAP } & \text { Glial fibrillary acidic protein } \\ \text { GS } & \text { Glutamine synthetase } \\ \text { HE } & \text { Haematoxylin-eosin } \\ \text { IB4 } & \text { Isolectin-B4 } \\ \text { IBA1 } & \text { Ionised calcium-binding adapter molecule 1 } \\ \text { INSR } & \text { Insulin receptor } \\ \text { K } & \text { Inward rectifier K }{ }^{+} \text {channel } \\ \text { MP } & \text { Mononuclear phagocyte } \\ \text { NG2 } & \text { Neuroglial antigen 2 } \\ \text { ONL } & \text { Outer nuclear layer } \\ \text { RAS } & \text { Renin-angiotensin system } \\ \text { RGC } & \text { Retinal ganglion cell } \\ \text { RPE } & \text { Retinal pigment epithelium } \\ \text { shRA } & \text { Small hairpin RNA } \\ \text { STZ } & \text { Streptozotocin } \\ \text { WT } & \text { Wild-type } \\ \text { ZO-1 } & \text { Zonula occludens 1 }\end{array}$

\section{Introduction}

Diabetic retinopathy is a major cause of blindness in the working-age population and is responsible for almost $20 \%$ of cases of vision loss in the western hemisphere [1]. Of those suffering from type 2 diabetes, 50-80\% develop retinopathy within 20 years of diagnosis $[2,3]$. Retinopathy prevalence increases with the duration of diabetes, the need for insulin injections, high $\mathrm{HbA}_{1 \mathrm{c}}$ levels and concomitant hypertension [4]. The mechanisms underlying the pathology that ultimately leads to vision loss are still poorly understood and current therapies address symptoms rather than causative factors. Thus, there is a need to further elucidate the pathogenesis of diabetic retinopathy.

The streptozotocin (STZ)-induced rodent model of type 1 diabetes mellitus is most often used to mimic diabetic retinopathy. In mice, astrocyte activation, retinal ganglion cell (RGC) death, vascular leakage and leucostasis are present after 8 weeks of diabetes [5], whilst rats are more susceptible to diabetes induction and this phenotype develops earlier [5-8]. However, compared with clinical observations in humans with type 1 diabetes, the diabetic vascular phenotype is rather weak in rodents. Moreover, the literature concerning animal models of type 2 diabetes is more sparse than for type 1 diabetes; the $d b / d b$ mouse, which develops diabetes due to a leptin receptor deficiency [9], is the most prominent model of type 2 diabetes. The few rat models available for experimental type 2 diabetes are not ideal since their disease progression is very slow and they cannot reliably reproduce the retinopathy phenotype [5].

We recently generated a novel model for type 2 diabetes [10]; the TetO transgenic rat carries a tetracycline-inducible small hairpin RNA (shRNA) expression system targeting the insulin receptor (INSR). Chronic doxycycline administration leads to hyperglycaemia and hyperinsulinaemia, caused by systemic inhibition of INSR expression and signalling, emulating type 2 diabetes $[10,11]$. Activation of the reninangiotensin system (RAS), mainly by its major effector peptide angiotensin-II and its receptor, angiotensin-II receptor 1 (AT1), has been shown to play an important role in the pathophysiology of diabetic nephropathy, neuropathy and retinopathy $[12,13]$. Evidence comes mainly from clinical intervention studies [14-16]; RAS inhibition partly and selectively ameliorated diabetes-induced changes [16] or reduced the incidence of diabetic retinopathy in patients [15]. The underlying mechanisms, however, have not been systematically explored.

The main purpose of this study was to test the suitability of TetO rats as a new model for diabetic retinopathy in type 2 diabetes and to investigate the ability of AT1 blockers (ARBs) to reverse diabetes-induced retinal damage.

\section{Methods}

Animals Four-month-old NTac:SD-Tg(H1/tetO-RNAi:Insr) 29Bdr rats (Max Delbrück Center, Berlin, Germany), here referred to as TetO rats, were used in the experiments. Male $(n=5)$ and female $(n=20)$ rats received $2 \mathrm{mg} /(\mathrm{kg}$ body weight) of doxycycline in their drinking water until they were considered to be diabetic (blood glucose $>16.65 \mathrm{mmol} / \mathrm{l}$ after 7-8 days of initial induction). Thereafter, the doxycycline dose was adapted to maintain blood glucose levels at $16.5-17 \mathrm{mmol} / \mathrm{l}$ for $4-5$ weeks. Blood glucose levels were monitored regularly using an Accu Chek Sensor (Roche, Mannheim, Germany). Age-matched doxycycline-fed Sprague-Dawley rats (Crl:SD; Charles River, Sulzfeld, Germany) served as wild-type (WT) controls $(n=20)$. In male rats, oral treatment with the ARB, losartan potassium (Sigma, Seelze, Germany) was started at the onset of diabetes $\left(10 \mathrm{mg} \mathrm{kg}^{-1} \mathrm{day}^{-1}\right)$. Rats were maintained in individually ventilated cages (Tecniplast, Buguggiate, Italy) under standardised conditions $\left(21^{\circ} \mathrm{C}, 65 \%\right.$ humidity, artificial $12 \mathrm{~h}$ 
light/dark cycle) with free access to standard chow $(0.25 \%$ $\mathrm{wt} / \mathrm{wt}$ sodium; SSNIFF, Soest, Germany) and drinking water.

All experiments were conducted in compliance with the ARVO Statement for the Use of Animals in Ophthalmic and Vision Research and were approved by governmental authorities (Landesamt für Gesundheit und Soziales [LAGeSo], Berlin, Germany). Samples were not randomised. Only TetO rats with blood glucose levels above $16.65 \mathrm{mmol} / \mathrm{l}$ were included in the diabetic group of the study.

ELISA and western blot For determination of serum insulin levels in WT and TetO rats, an insulin ELISA kit (Mercodia, Uppsala, Sweden) was used according to the manufacturer's instructions.

INSR expression in the retina was quantified by western blot according to standard protocols, using primary antibodies against INSR (Sigma) and $\beta$-actin (Sigma) and horseradish peroxidase-conjugated secondary antibodies (GE Healthcare, Little Chalfont, UK). Proteins were visualised using Clarity ECL Western Blotting Substrate (Bio-Rad, Hercules, CA, USA). Densitometry analysis of INSR expression was performed using Quantity One 1-D Analysis Software (Bio-Rad). A more detailed description of the western blot method is provided in the electronic supplementary material (ESM) Methods and ESM Table 1.

Immunohistochemical analysis in paraffin sections Rat eyes were enucleated post-mortem, fixed in $4 \% \mathrm{wt} / \mathrm{vol}$. paraformaldehyde and embedded in paraffin. Deparaffinised sections of $5 \mu \mathrm{m}$ thickness were subjected to different standard antigen retrieval treatments, blocked in $5 \% \mathrm{wt} / \mathrm{vol}$. BSA and subsequently incubated with primary antibody in $0.8 \% \mathrm{wt} / \mathrm{vol}$. BSA overnight, at $4^{\circ} \mathrm{C}$, followed by speciesappropriate secondary antibodies. Microscope imaging was performed with an Axio Imager M2 fluorescence microscope (Zeiss, Jena, Germany) using ZEN Lite 2012 software (Zeiss). Vessels were analysed detecting collagen IV (2150-0140, Bio-Rad). Müller cells were evaluated using antibodies against glutamine synthetase (GS; ab49873, Abcam, Cambridge, UK), glial fibrillary acidic protein (GFAP; Z0334, DAKO, Hamburg, Germany) and inward rectifier $\mathrm{K}+$ channel $\left(\mathrm{K}_{\mathrm{ir}}\right) 4.1$ (APC-035, Alomone, Jerusalem, Israel). Quantification was done manually using 4-5 representative visual fields per animal and reported as number of (GS)-positive cells per $1 \mathrm{~mm}$. Albumin (A110-134A, Bethyl, Montgomery, TX, USA) leakage was quantified by measuring colour pixels $/ \mathrm{mm}^{2}$ after eliminating intravascular albumin staining (2-6 representative visual fields per animal). Pixel analysis was carried out using ImageJ 1.50 (NIH, Bethesda, MD, USA). Additional information about antibodies employed can be found in ESM Table 1. Primary antibodies were tested and validated in previous published studies. All secondary antibodies were validated using negative controls for primary antibodies in each experiment.

Quantification of retinal ganglion cells and outer nuclear layer nuclei Retinal sagittal sections were stained with haematoxylin-eosin (HE) and quantitative analysis of RGCs was performed exclusively in a series of cross-sections that captured the optic nerve. Counting of RGCs was carried out manually in a blinded manner. RGCs were identified by size, shape and granularity. Ratios of counted RGCs were normalised to $100 \mu \mathrm{m}$. Quantitative analysis of outer nuclear layer (ONL) nuclei was conducted in the same manner, with ratios of counted nuclei also normalised to $100 \mu \mathrm{m}^{2}$.

Fluorescein angiography Rats were subjected to pupil dilatation with phenylephrine and tropicamide eye drops (Apotheke der Charité, Berlin, Germany). After 10 min, they were shortly sedated with isoflurane (Forene, Abbvie, Ludwigshafen, Germany) and anaesthetised by i.p. injection of ketamine hydrochloride $(60 \mathrm{mg} / \mathrm{kg}$; Actavis, Munich, Germany) and xylazine (Rompun, $9 \mathrm{mg} / \mathrm{kg}$; Bayer, Leverkusen, Germany). Spectralis HRA-OCT (Heidelberg Engineering, Heidelberg, Germany) was used in autofluorescence imaging (AF) mode for angiography (solid-state laser, wavelength $488 \mathrm{~nm}$ ). A 30D lens was employed to make the system suitable for rodents. To visualise blood vessels, fluorescein was injected (Fluorescein $10 \%$ wt $/ \mathrm{vol}$., $5 \mathrm{mg} / \mathrm{kg}$; Alcon, Freiburg, Germany). Images were recorded $10 \mathrm{~min}$ after injection and were analysed by Heidelberg Eye Explorer software 1.7.0.0 (Heidelberg Engineering).

Analysis in retinal whole mounts Enucleated eyes were punctured in the cornea and fixed in $4 \% \mathrm{wt} / \mathrm{vol}$. paraformaldehyde for $30 \mathrm{~min}$. Cornea, lens and vitreous were dissected and discarded. Four radial cuts were made at the eyecup to allow its flattening and the optic nerve was sectioned to separate the neural retina fraction from the sclera. Separately, both fractions were repeatedly washed in Tris-buffered saline and then incubated in a blocking solution containing $15 \% \mathrm{wt} / \mathrm{vol}$. of normal goat serum (DAKO). After antibody incubations, whole mounts were mounted onto glass slides and imaged with an LSM 510 confocal microscope (Zeiss), using ZEN Lite 2012 software (Zeiss). Primary antibodies were tested and validated in previous published studies. All secondary antibodies were validated using negative controls for primary antibodies in each experiment.

Ionised calcium-binding adapter molecule 1 (IBA1; 019-19741, Wako, Neuss, Germany) was employed to detect mononuclear phagocytes (MPs) [17]. Distinguishing deep and superficial retinal layers by adjusting the microscope focus, 
5-10 randomised pictures were taken per sample. Quantification was normalised to cells per $1 \mathrm{~mm}^{2}$. Activation status was determined based on phenotype criteria: ramified cells were considered 'surveying' and amoeboid cells, 'activated' [18].

Pericytes were detected with an antibody against neuroglial antigen 2 (NG2; AB5320C3, Millipore, Darmstadt, Germany) [19]. Quantification was performed using 5-10 representative image fields per sample. Ratios of cells were normalised to $1 \mathrm{~mm}^{2}$. Stenotic vessels were quantified as thoroughly described by Reichhart et al using Isolectin B4 (IB4; 121411, Life Technologies, Darmstadt, Germany) [20]. Additional information about antibodies can be found in ESM Table 1.

Quantitative PCR RNA isolation and real-time quantitative PCR (qPCR) was carried out according to the methods described previously by our group [20]. Briefly, the cornea and lens were removed and discarded from eyes of WT and TetO rats. RNA was isolated from remaining eye tissue using the RNeasy Mini Kit (Qiagen, Hilden, Germany) and converted to cDNA, prior to quantification of mRNA expression using the relative standard curve methods, using 18S RNA as an endogenous control. A more detailed description of the procedure is provided in ESM Methods. Primer sequences are listed in ESM Table 2.

Electroretinography analysis In brief, electroretinography (ERG) was performed in a Ganzfeld bowl (Roland Consult, Brandenburg, Germany) as described by Reichhart et al [20]. Monopolar contact lens electrodes served as recording electrodes and subcutaneous platin needles as reference electrodes. A flash series consisting of 10 steps from -4.0 $\log _{10} \mathrm{~cd} \times \mathrm{s} / \mathrm{m}^{2}$ to $0.48 \log _{10} \mathrm{~cd} \times \mathrm{s} / \mathrm{m}^{2}$ was applied. Oscillatory potentials were obtained with a flash intensity of $0.48 \log _{10} \mathrm{~cd} \times \mathrm{s} / \mathrm{m}^{2}$. The a-wave was recorded using flash energies of: 0.97 and $1.48 \log _{10} \mathrm{~cd} \times \mathrm{s} / \mathrm{m}^{2}$. The b-wave amplitude was measured from a-wave trough to $b$-wave peak. The $c$-wave amplitude was measured from the pre-stimulus baseline to the peak of the c-wave. Photopic ERG on light adapted animals was recorded using a series of flash energies of 0.7 and $1.3 \log _{10} \mathrm{~cd} \times \mathrm{s} / \mathrm{m}^{2}$. A more detailed description of the procedure is provided in ESM Methods.

Statistical analysis All experiments were repeated at least in triplicate and evaluation was carried out in a blinded fashion. ' $n$ ' refers to the number of rats. Results are expressed as means \pm SEM. Statistical differences were calculated using two-tailed ANOVA, and considered significant when $p<0.05$. The Kruskal-Wallis test for independent samples, with critical $p$ value $=0.05$, was applied for ERG data.

\section{Results}

INSR knockdown in the retina Since the tissue susceptibility for knockdown of the INSR in TetO rats is variable [10], the efficacy of the INSR knockdown in retina was verified once blood glucose and insulin levels in TetO rats were significantly increased (insulin: $5.209 \pm 0.022 \mu \mathrm{mol} / 1$ in TetO rats vs $0.286 \pm 0.919 \mu \mathrm{mol} / \mathrm{l}$ in WT rats; Fig. 1a). Immunohistochemical analysis of WT sagittal sections revealed the presence of INSR mainly in the ganglion cell layer (GCL) and in the ONL. Only a very weak signal was detected in the transgenic animals (ESM Fig. 1a). Total gene and protein levels of INSR in retina were significantly reduced in TetO rats compared with WT rats, resulting in approximately a twofold reduction in the expression of Insr mRNA and a $\sim 40 \%$ reduction in the expression of INSR protein in TetO rats (Fig. 1b, c and ESM Fig. 1b).

AT1 in the diabetic retina Gene expression levels of At1 (also known as Agtrla) were significantly increased in the TetO retina compared with the WT retina (Fig. 1d). Staining with an antibody against activated AT1 showed significantly higher protein expression levels in the inner retina of TetO vs WT rats (Fig. 1e, ESM Fig. 1c).

Structural analysis of the retina Retinal structure was analysed ex vivo in sagittal sections stained with HE. TetO rats did not show any structural disruption of the retinal layers. ONL nuclei density was similar between the groups (ESM Fig. 1d). The GCL, however, revealed a significant reduction in the number of ganglion cells in the transgenic rats $(3.52 \pm 0.13$ cells $/ 100 \mu \mathrm{m})$ compared with WT rats (4.75 \pm 0.06 cells $/ 100 \mu \mathrm{m}$; Fig. 1f). Losartan treatment prevented the diabetes-induced loss of RGCs in TetO rats $(5.33 \pm 0.15$ cells $/ 100 \mu \mathrm{m}$; Fig. $1 \mathrm{f})$.

Analysis of the retinal vasculature Fundus angiography revealed no apparent changes in vascular morphology in either group (Fig. 2a). Nevertheless, ex vivo analysis revealed several pathological characteristics in TetO retinas. Transgenic rats showed significantly more stenotic vessels compared with WT rats in mid-peripheral areas of the retina (Fig. 2b, c). The presence of veins with abnormal vessel calibre in the deep microvascular plexus, located in mid-peripheral areas of the retina, was remarkable in both treated and untreated TetO rats (Fig. 2b). Losartan treatment did not ameliorate the diabetes-induced vascular pathologies (Fig. 2b, c).

Pericyte coverage in TetO rats was significantly reduced in the diabetic retina, compared with WT rats (Fig. 2d). Losartan treatment led to partial recovery of pericyte coverage, although this did not reach statistical significance. 
Fig. 1 INSR knockdown and structural alterations in the retina of TetO rats. (a) Insulin levels $(n=4-5)$ in serum measured by ELISA. (b) Insr mRNA expression in retina (fold normalised to $18 \mathrm{~S}, n=9-10$ ). (c) Relative INSR expression determined by western blot (fold normalised to INSR expression in WT rats, $n=6-7$ ). (d) At 1 mRNA expression in retina (fold normalised to $18 \mathrm{~S}, n=9-10$ ). (e) Quantification of colour pixels of activated AT1 (actAT1) per area given in arbitrary units (AU) $(n=4)$. (f) Representative retinal sagittal sections stained with HE. Scale bar, $50 \mu \mathrm{m}$. Bar chart shows the number of RGCs normalised to $100 \mu \mathrm{m}^{2}$ ( $\left.n=5-7\right)$. Los, losartan. INL, inner nuclear layer; OS, outer segments. ${ }^{*} p<0.05$, $* * * p<0.001$ a
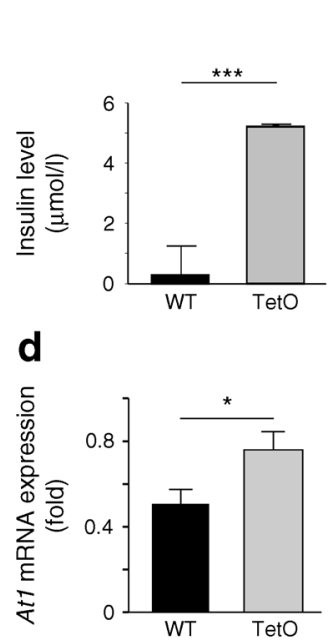

f

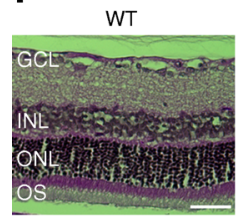

b

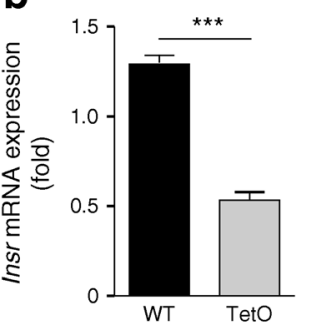

C
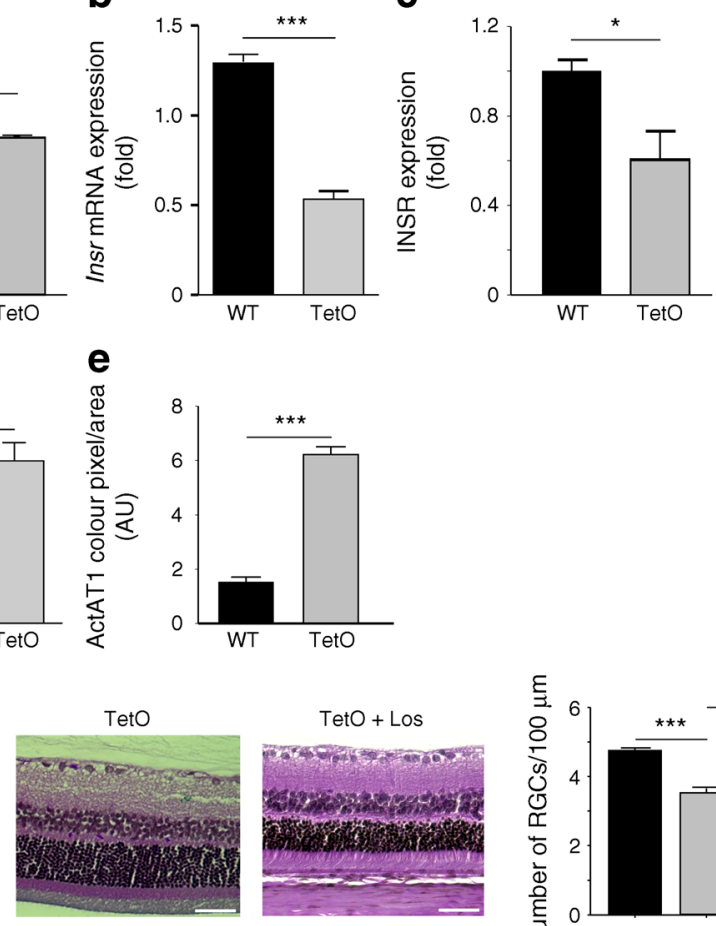

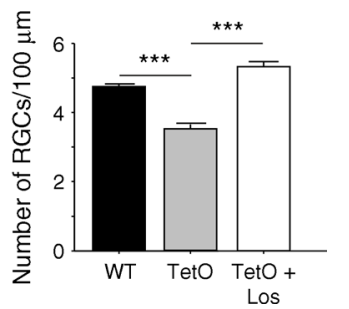

Collagen IV, a major component of the basal membrane, showed no difference in expression in sagittal sections when comparing the groups (Fig. 2e).

Disruption of the blood-retina barrier Extravasation of albumin is an indicator of functional breakdown of the blood-retina barrier (BRB). Albumin leakage was detected in TetO rats (both losartan-treated and untreated), whereas no leakage was found in WT rats (Fig. 3a).

Evidence of alterations in the retinal pigment epithelium (RPE; outer BRB) was found in sclera whole mounts stained against the tight-junction proteins, zonula occludens-1 (ZO-1) and claudin-1. Whereas claudin-1 expression was unaltered, ZO-1 protein expression became patchy and disorganised, and the geometry and cell size of RPE in TetO presented a higher degree of variability (ESM Fig. 2).

Müller cell pathology Glutamine synthetase (GS) is involved in transmitter recycling [21] and was employed to identify Müller cells in sagittal sections. No difference in the number of GS-positive cells was found when comparing WT, TetO and losartan-treated TetO rats (Fig. 3b).

Overexpression of glial fibrillary acidic protein (GFAP) is one of the first histological findings in retinas affected by diabetes [22-25]. In retinal sagittal sections of both transgenic rats and control WT rats, a GFAP signal was detected in astrocytes. Only untreated TetO rats showed increased anti-GFAP reactivity in Müller cells (Fig. 3c). The major potassium channel in Müller cells is the inward rectifier
$\mathrm{K}^{+}$channel $\left(\mathrm{K}_{\mathrm{ir}}\right) 4.1$, which drives the elimination of water from the retina [26-29]. A $K_{\text {ir }} 4.1$ signal in sagittal sections of the WT rats was found exclusively in Müller cells, while in the TetO rats $\mathrm{K}_{\mathrm{ir}} 4.1$ was delocalised and ubiquitously expressed among the retina (Fig. 3d). Losartan treatment prevented the diabetes-related changes in GFAP and $\mathrm{K}_{\mathrm{ir}} 4.1$, with localisation of these proteins comparable with that in the WT rats (Fig. 3c, d).

MPs and inflammation To examine general inflammation in the retina, a panel of markers was assessed by qPCR. Compared with WT rats, untreated TetO rats had significantly higher levels of mRNA expression for Tnfa (also known as Tnf), Cd18 (also known as Itgb2), Ccl2 and Icam1. Vcam1 and $T g f b$ (also known as $T g f b l$ ) expression was not significantly altered (Fig. 4a-f).

MP activation status was determined by phenotype, as described in the Methods. We distinguished between the superficial retina (composed of the GCL and the inner plexiform layer [IPL]) and the deep retina (containing the microglia in the outer retinal layer). Most MPs were found in the superficial retina rather than in the deep retina. WT rat retinas displayed low rates of activation, while, in comparison, untreated TetO rat retinas presented similar rates of surveying MPs but significantly higher rates of activated cells (Fig. 4g, h). In the superficial retina, a slight, although non-significant, decrease in both surveying and activated MPs was observed in losartan-treated TetO rats compared with untreated TetO (Fig. 4g), yet the overall amount of 
Fig. 2 Retinal vascular phenotype. (a) Representative fluorescence angiography of WT and $\mathrm{TetO}$ rat retinas. (b) Retinal whole mounts stained against IB4 to detect vessels in the superficial and deep plexuses. (c) Stenotic vessels (arrows) can be clearly identified in TetO rat retinas. Bar chart shows quantification of stenotic vessels $/ \mathrm{mm}^{2}$ in the superficial and deep retina $(n=4-5)$. (d) Retinal whole mounts stained against NG2 (red) and IB4 (green) in the superficial retina. Bar chart shows the pericyte coverage $(n=3-4)$. For (c) and (d), black bars, WT; grey bars, TetO; white bars, TetO + losartan. (e) Co-staining of retinal sections against collagen IV (Coll IV; red) and IB4 (green). Nuclei were counterstained with DAPI (blue). Scale bar, $50 \mu \mathrm{m}$. Los, losartan; INL, inner nuclear layer; OS, outer segments. * $p<0.05$, $* * p<0.01$ a
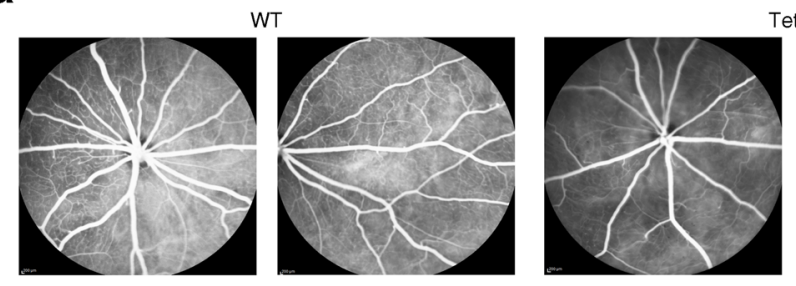

Teto

b
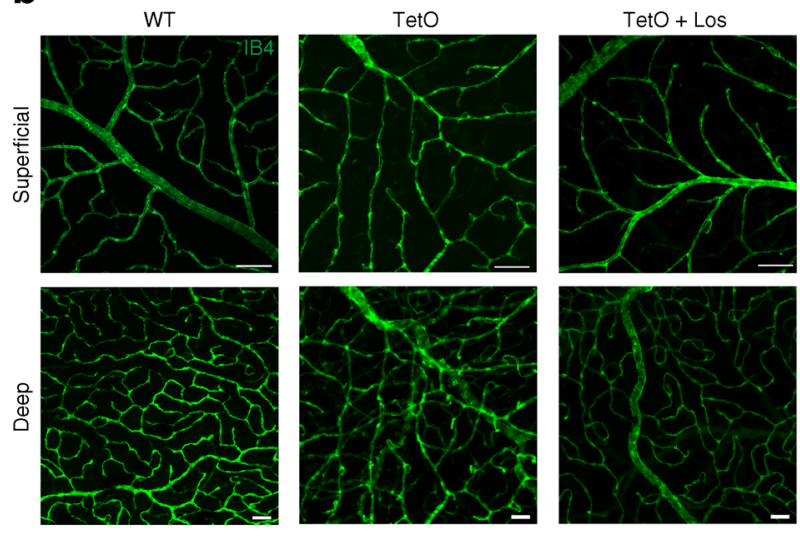

C
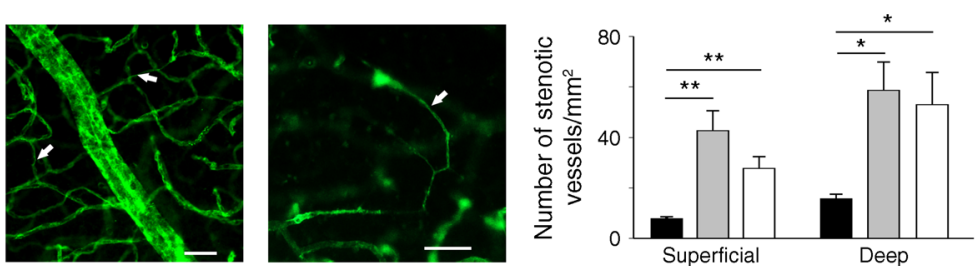

d
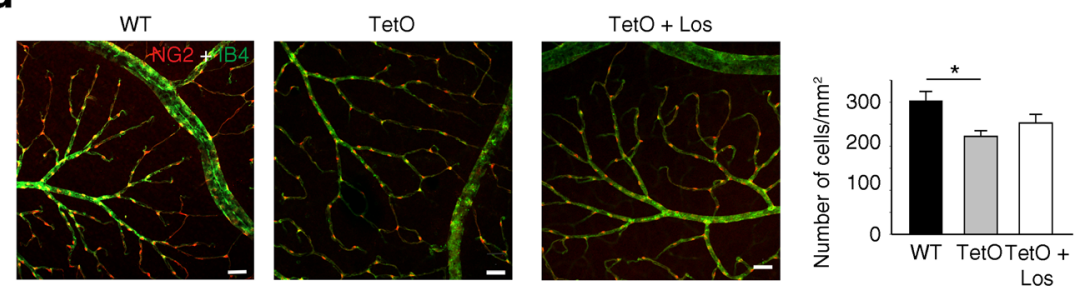

e
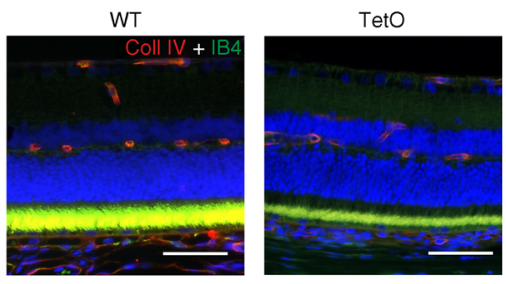

amoeboid MPs in the superficial retina was much higher (although not significantly) in losartan-treated TetO rats than in WT rats (Fig. 4g).

Functional changes detected by ERG We assessed retinal function by ERG under scotopic and photopic conditions. Scotopic ERG reflects rod function whereas photopic ERG is related to cones. A significant reduction in scotopic a- and b-waves was found in untreated TetO vs WT rat retinas (Fig. 5a, b). The b/a-wave ratio was elevated in TetO rats vs.
WT, suggesting a predominant rod photoreceptor function loss (Fig. 5d). Furthermore, the c-wave, reflecting the increase in transepithelial potential light-induced hyperpolarisation of the RPE apical membrane, was also significantly reduced in TetO compared with WT (Fig. 5c). The a/c-wave ratio, however, remained unchanged (data not shown), suggesting that the c-wave reduction is a consequence of the a-wave reduction and, thus, of the functional loss of photoreceptors.

As the photopic a-wave in rodents is very small, assessment of cone function was based on photopic b-wave 
Fig. 3 Leakage and Müller cell pathology. (a) Staining of retinal sagittal sections against albumin (red) illustrates extravasation in $\mathrm{TetO}$ and $\mathrm{TetO}+$ losartan) (arrows). Bar chart shows the percentage of extravasated albumin colour pixels in relation to the total retinal field $(n=3)$. (b) Staining of retinal sections against GS (yellow). Bar chart shows quantification of GSpositive Müller cells per mm $(n=3-5)$. (c, d) Staining of retinal sections against (c) GFAP (green) and (d) $\mathrm{K}_{\mathrm{ir}} 4.1$ (red). Nuclei were counterstained with DAPI (blue). Scale bar, $50 \mu \mathrm{m}$. Los, losartan; INL, inner nuclear layer; OS, outer segments. $* * p<0.01$
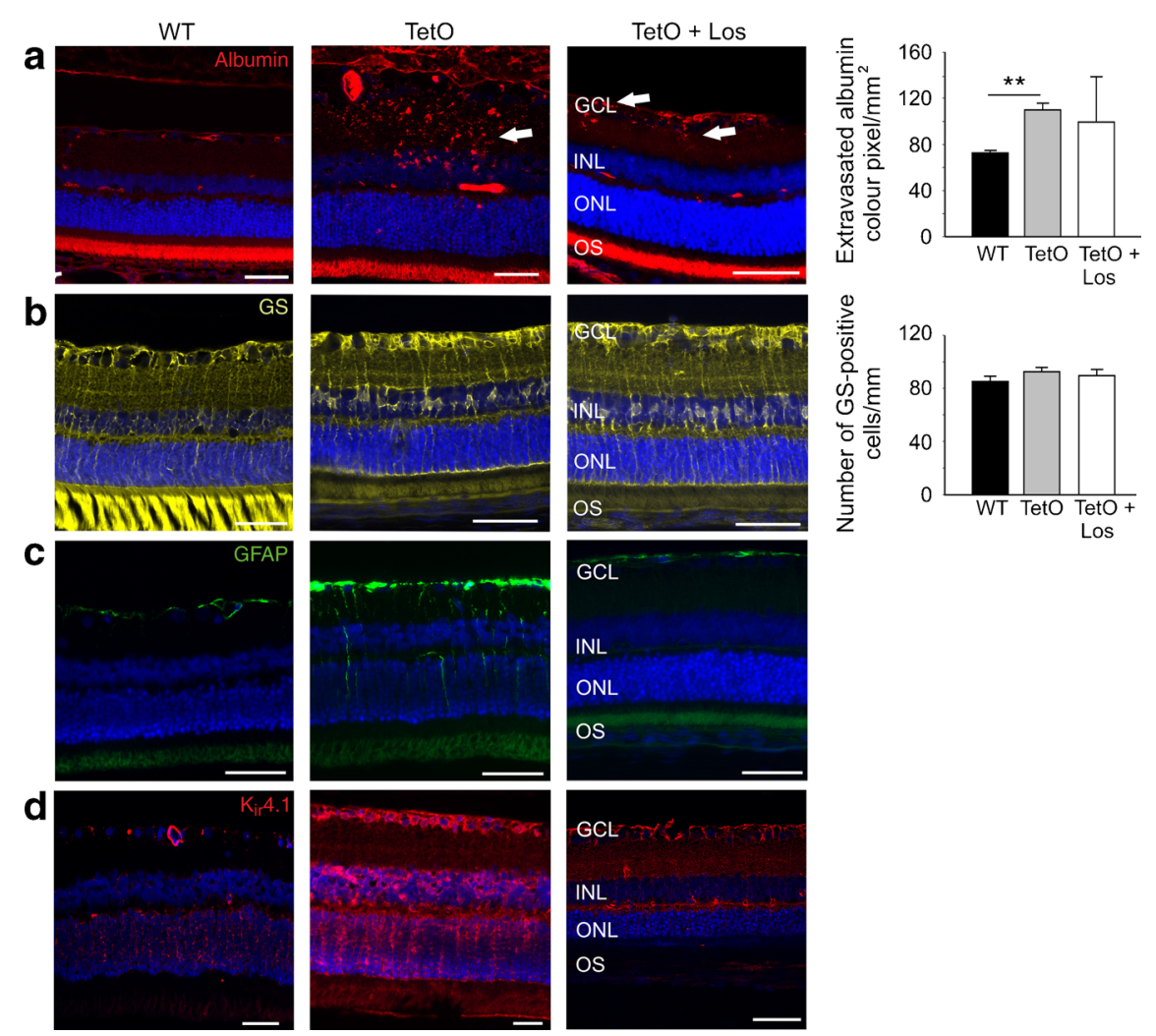

TetO rats present both hyperinsulinaemia and hyperglycaemia, defining type 2 diabetes and mimicking insulin resistance in humans. The model shows an early onset of hyperglycaemia with subsequent early neurovascular effects. Given the spectrum of mechanisms underlying human diabetic retinopathy (e.g. hyperglycaemia, hyperinsulinaemia and altered lipid metabolism) the TetO rat is potentially a very valuable translational model for insulin resistance in type 2 diabetes.

Comparing TetO rats with the most common diabetes model, the STZ-induced rodent model of type 1 diabetes, we also find many of the STZ-induced early changes to be present in our short-term diabetes model. Breakdown of the BRB, as shown by albumin extravasation or by loss of ZO-1 expression in the RPE in TetO, has been reported in STZ rats after 2 weeks [5]. Additionally, reactive gliosis, as we have shown in the TetO rat, was also observed in STZ mice at a similar time point [5]. In rats, however, there was a rather contrary effect: a reduction in the number of astrocytes after 2-6 weeks [5, 25]. Also, an increase in GFAP expression and delocalisation of $\mathrm{K}_{\mathrm{ir}} 4.1$ in Müller cells was detected much later in the STZ model than in the TetO model [27, 30, 31]. Stenotic capillaries, found as an early feature in the TetO model, were detected in the STZ model only as a late finding after 6-9 months of diabetes [5]. RGC loss and visual function changes, which is reported in humans even before detectable vascular impairment [32], were also found in TetO rats within 4-5 weeks, whereas STZ model animals only shows these alterations in very late stages [5]. 
Fig. 4 Inflammation in the retina. (a-f) Bar charts represent the expression of the genes indicated $(n=10-11)$. (g, h) Staining of representative whole mounts against IBA1 (red) in the (g) superficial and (h) deep retina. Scale bar, $100 \mu \mathrm{m}$. Bar charts show quantification of MPs in whole mounts $(n=2-6)$. Black bars, WT; grey bars, TetO; white bars, TetO + losartan. Amoe, amoeboid activated MPs; Los, losartan; Ram, ramified surveying MPs. $* p<0.05, * * p<0.01$, $* * * p<0.001$
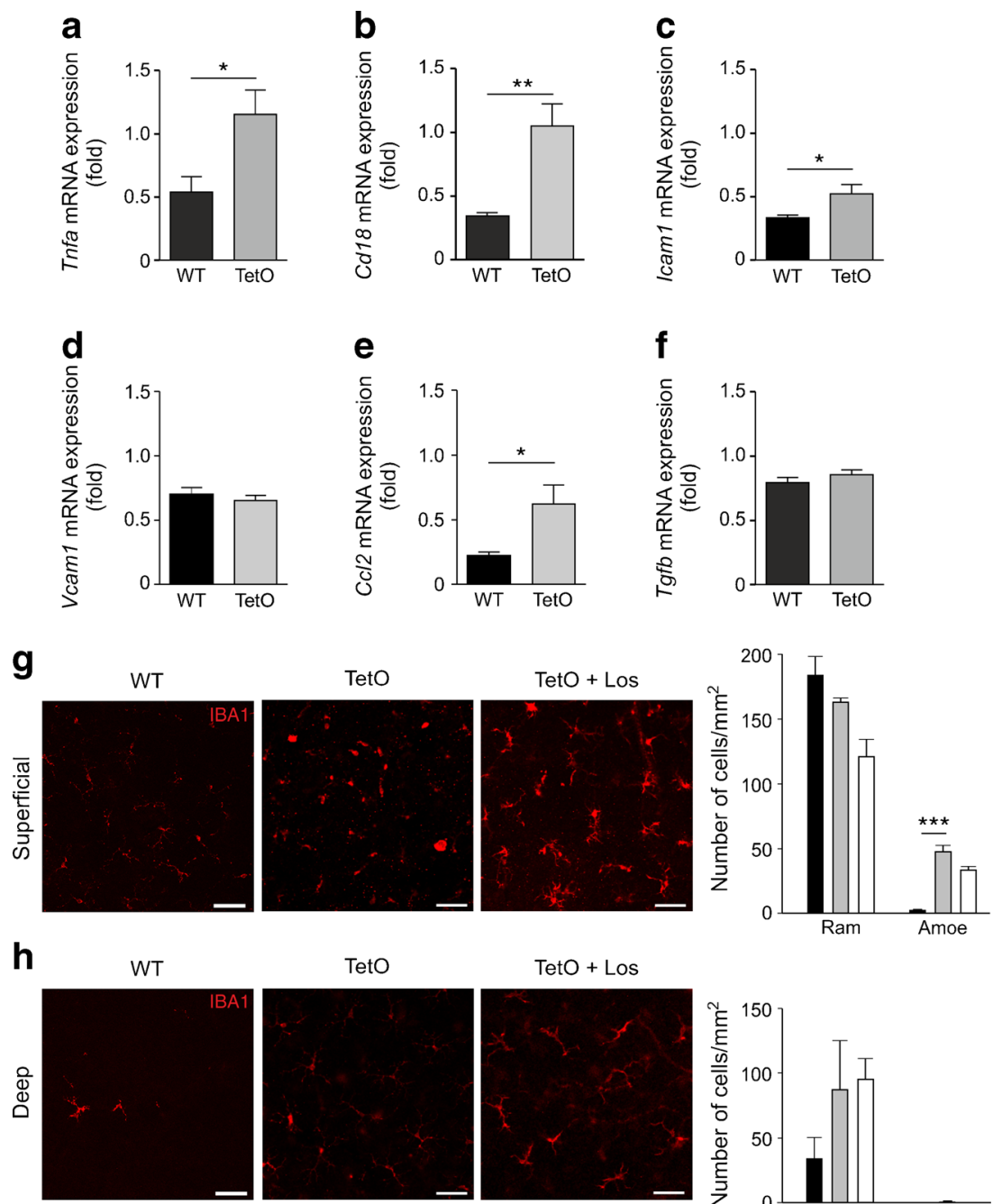

TetO

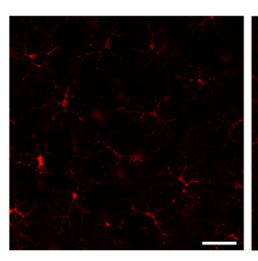

TetO + Los
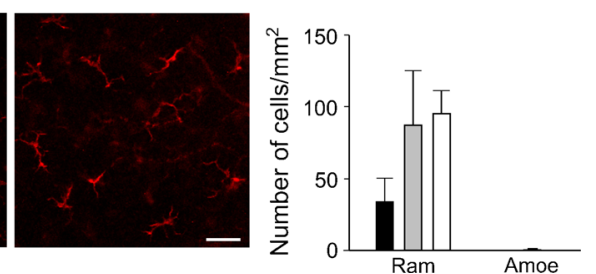

In the context of a pro-inflammatory scenario leading to diabetes-related changes, MP activation appears early in the course of the disease, before the onset of overt neuronal cell death $[33,34]$. A general increase in isolectin-B4 (IB4)-positive microglia cells was detected in STZ rats 4 weeks after the onset of type 1 diabetes. However, this marker that does not reliably cover the population of microglia cells in the retina [25]. A morphological switch towards the active amoeboid phenotype was also found in STZ rats, but this was observed as late as 20 weeks [5]. Thus, in the present study, in TetO rats (even in the early stages of diabetes) we found diabetic features, previously identified in both rodent models and humans, that are not reproduced efficiently, either chronologically or phenotypically by STZ-treatment of rats. Furthermore, interesting additional TetO properties were detected in this study, such as prominent veins in the deep plexus and changes in RPE cell morphology. These properties require further examination in the context of diabetes-related changes.

The few existing type 2 diabetes animal models reveal diabetic hallmarks only up to a certain extent, even after long periods of the disease. Also, they do not present with the combination of high glucose and insulin levels, such as demonstrated in the TetO rat. $d b / d b$ mice develop diabetes due to a mutation of the leptin receptor [9] and display RGC loss and thinning of the retina as early features [34]. This is comparable with our model. However, other diabetic hallmarks, such as vascular leakage and glial activation, which were detected after a few weeks of diabetes in the TetO rat, develop relatively late in $d b / d b$ mice [35]. Considering rat models of type 2 diabetes, such as the popular Wistar Bonn/Kobori rats, Otsuka Long-Evans Tokushima fatty rats or Goto-Kakizaki rats, the focus of research has been concentrated on vascular hallmarks, present only after more than 6 months of diabetes [5]. Neurodegeneration and ERG, which were important features of our study in TetO rats, were not assessed in these models.

Our data revealed AT1 activation in RGCs, endothelial cells and possibly in Müller cells in the TetO retina, suggesting a role for RAS activation mainly in the inner retina. There is emerging evidence that RAS is involved in the pathogenesis 

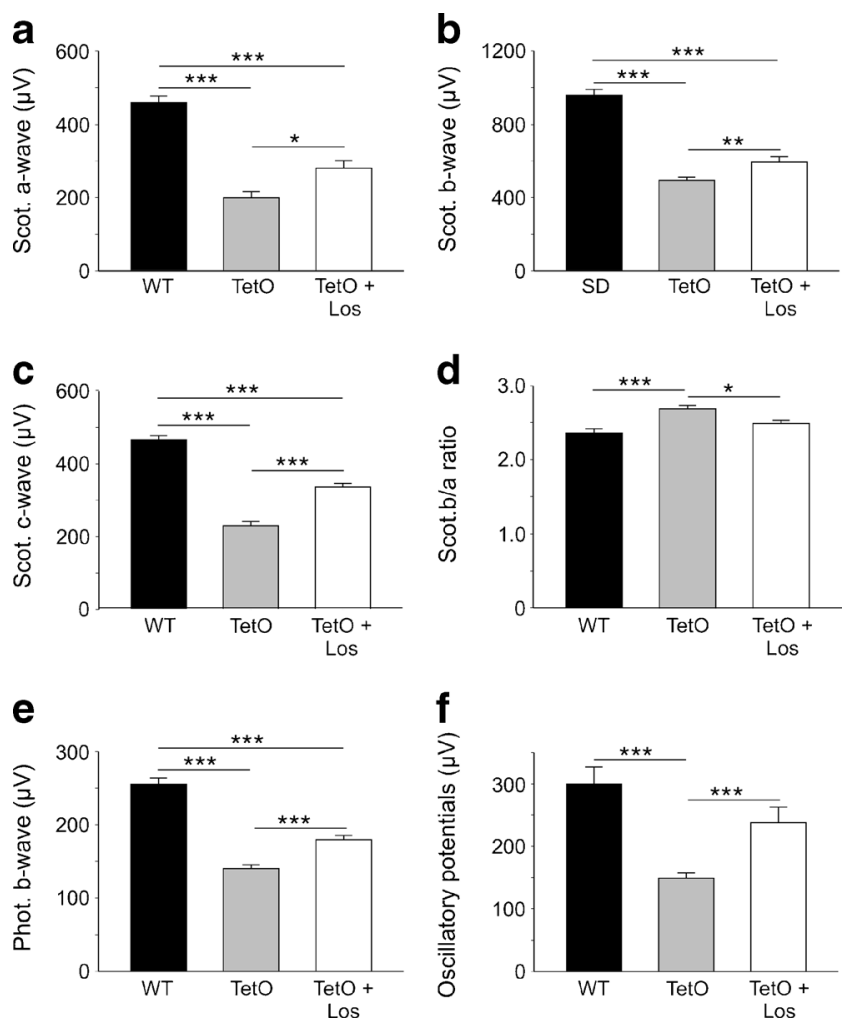

Fig. 5 Neurodegeneration and ERG changes. Bar charts illustrating: (a) scotopic a-wave, (b) scotopic b-wave, (c) scotopic c-wave and (d) scotopic b/a ratio, determined by ERG in retinas of WT rats, TetO rats and TetO rats treated with losartan. (e) Bar chart illustrating photopic b-wave. (f) Bar chart illustrating oscillatory potentials. $n=10$ for WT, $n=7$ for TetO $n=5$ for TetO + losartan. Los, losartan; Phot. Photopic; Scot., scotopic. $* p<0.05$, * $p<0.01, * * * p<0.001$

of diabetes via promotion of endothelial apoptosis and remodelling, inflammation or oxidative stress [36-38]. In diabetic organ damage, AT1 is the main receptor mediating the pathological effect. ARBs have been shown to be beneficial in clinical cases of diabetic retinopathy and nephropathy [39]. The Diabetic Retinopathy Candesartan Trials (DIRECT) [14, 15], using the ARB candesartan in type 1 and 2 diabetes, showed a clear trend towards less severe retinopathy with RAS blockade. A smaller trial, the Renin-Angiotensin System Study (RASS), reported that RAS blockade with both the ARB losartan and the angiotensin-converting enzyme (ACE) inhibitor, enalapril, resulted in reduced retinopathy progression in type 1 diabetes [16]. Experimental evidence concerning the effect of ARBs is sparse. Application of the ACE inhibitor, perindopril, reduced diabetes-induced hyperpermeability of the retinal vasculature after 24 weeks in the STZ model [40], and the same drug improved photoreceptor function [41].

We systematically evaluated the influence of the ARB, losartan, on neurovascular structures in the TetO rat. Its impact was only significant on the neuronal components analysed, showing decreased gliosis, RGC recovery and improved ERG variables. An influence on vasculature was not detectable, although the decrease in inflammation caused by losartan was close to reaching statistical significance.

In summary, the TetO rat exhibits early vascular and neuronal features of experimental diabetic retinopathy to a more complete and clinically relevant extent than currently used rodent models of diabetes. The fact that ARB treatment resulted in amelioration of characteristics more related to neurodegeneration than to vascular remodelling and inflammation emphasises the relevance of the RAS as a pathological mechanism in diabetic retinopathy and proves the suitability of the TetO model for interventional studies.

The TetO model permits modulation of the degree of hyperinsulinaemia and hyperglycaemia, enabling reproduction of clinically relevant situations, such as glucose concentration fluctuations, which can trigger progression of diabetic retinopathy $[42,43]$. This model could also reproduce characteristics closer to those found in proliferative diabetic retinopathy or diabetic macular oedema, as well as other long-term effects, within a considerably shorter time than in other rodent models. By not focusing on separate factors, such as high glucose (e.g. as in STZ models) or high insulin levels, but by taking into account their interplay, the TetO model is more valuable in the research of pharmaceutical interventions.

Acknowledgements We thank G. Fels (Charité-Universitätsmedizin Berlin, Germany), K. Oberländer (Charité-Universitätsmedizin Berlin, Germany), J. Meisel (Max Delbrück Center, Germany) and A. Schiche (Max Delbrück Center, Germany) for technical support and C. Corkhill (Charité-Universitätsmedizin Berlin, Germany) for language editing.

Funding Funding was provided by a Marie Curie grant in the framework of the REVAMMAD ITN (Project number 316990) and an unrestricted research grant by Novartis. MG is a participant in the BIH-Charite Clinical Scientist Program funded by the Berlin Institute of Health (BIH) and the Charite. The study sponsor was not involved in the design of the study; the collection, analysis, and interpretation of data; writing the report; or the decision to submit the report for publication.

Duality of interest The authors declare that there is no duality of interest associated with this manuscript.

Contribution statement Experiments were designed by NR, SC-G, MB, $\mathrm{OS}, \mathrm{RD}$ and $\mathrm{AMJ}$ and data was acquired and analysed by NR, SC-G, NH, MG, SS, CH, AR, NK and NA. The manuscript was written by NR, SC-G, OS, NA, MB, RD and AMJ and all authors revised and approved the final version for publication. The guarantor of this work is AMJ.

\section{References}

1. Resnikoff S, Pascolini D, Etya ale D et al (2004) Global data on visual impairment in the year 2002. Bull World Health Organ 82:844-851

2. Klein R, Klein BE, Moss SE (1989) The Wisconsin epidemiological study of diabetic retinopathy: a review. Diabetes Metab Rev 5:559-570 
3. Romero-Aroca P, Sagarra-Alamo R, Basora-Gallisa J, BasoraGallisa T, Baget-Bernaldiz M, Bautista-Perez A (2010) Prospective comparison of two methods of screening for diabetic retinopathy by nonmydriatic fundus camera. Clin Ophthalmol 4: 1481-1488

4. Yau JW, Rogers SL, Kawasaki R et al (2012) Global prevalence and major risk factors of diabetic retinopathy. Diabetes Care 35:556564

5. Lai AK, Lo AC (2013) Animal models of diabetic retinopathy: summary and comparison. J Diabetes Res 2013:106594

6. Joussen AM, Doehmen S, Le ML et al (2009) TNF- $\alpha$ mediated apoptosis plays an important role in the development of early diabetic retinopathy and long-term histopathological alterations. Mol Vis $15: 1418-1428$

7. Joussen AM, Poulaki V, Le ML et al (2004) A central role for inflammation in the pathogenesis of diabetic retinopathy. FASEB J 18:1450-1452

8. Joussen AM, Poulaki V, Mitsiades N et al (2003) Suppression of Fas-FasL-induced endothelial cell apoptosis prevents diabetic blood-retinal barrier breakdown in a model of streptozotocininduced diabetes. FASEB J 17:76-78

9. Hummel KP, Dickie MM, Coleman DL (1966) Diabetes, a new mutation in the mouse. Science 153:1127-1128

10. Kotnik K, Popova E, Todiras M et al (2009) Inducible transgenic rat model for diabetes mellitus based on shRNA-mediated gene knockdown. PLoS One 4, e5124

11. Santos SH, Giani JF, Burghi V et al (2014) Oral administration of angiotensin-(1-7) ameliorates type 2 diabetes in rats. J Mol Med 92: 255-265

12. Wilkinson-Berka JL (2004) Diabetes and retinal vascular disorders: role of the renin-angiotensin system. Expert Rev Mol Med 6:1-18

13. Kobori H, Kamiyama M, Harrison-Bernard LM, Navar LG (2013) Cardinal role of the intrarenal renin-angiotensin system in the pathogenesis of diabetic nephropathy. J Investig Med 61:256-264

14. Sjolie AK, Klein R, Porta M et al (2008) Effect of candesartan on progression and regression of retinopathy in type 2 diabetes (DIRECT-Protect 2): a randomised placebo-controlled trial. Lancet 372:1385-1393

15. Chaturvedi N, Porta M, Klein R et al (2008) Effect of candesartan on prevention (DIRECT-Prevent 1) and progression (DIRECTProtect 1) of retinopathy in type 1 diabetes: randomised, placebocontrolled trials. Lancet 372:1394-1402

16. Harindhanavudhi T, Mauer M, Klein R et al (2011) Benefits of renin-angiotensin blockade on retinopathy in type 1 diabetes vary with glycemic control. Diabetes Care 34:1838-1842

17. Ito D, Imai Y, Ohsawa K, Nakajima K, Fukuuchi Y, Kohsaka S (1998) Microglia-specific localisation of a novel calcium binding protein, Iba1. Brain Res Mol Brain Res 57:1-9

18. Langmann T (2007) Microglia activation in retinal degeneration. J Leukoc Biol 81:1345-1351

19. Hughes S, Chan-Ling T (2004) Characterization of smooth muscle cell and pericyte differentiation in the rat retina in vivo. Invest Ophthalmol Vis Sci 45:2795-2806

20. Reichhart N, Haase N, Crespo-Garcia S et al (2016) Hypertensive retinopathy in a transgenic angiotensin-based model. Clin Sci 130: 1075-1088

21. Pow DV, Robinson SR (1994) Glutamate in some retinal neurons is derived solely from glia. Neuroscience 60:355-366

22. Barber AJ, Antonetti DA, Gardner TW (2000) Altered expression of retinal occludin and glial fibrillary acidic protein in experimental diabetes. The Penn State Retina Research Group. Invest Ophthalmol Vis Sci 41:3561-3568
23. Lieth E, Barber AJ, Xu B et al (1998) Glial reactivity and impaired glutamate metabolism in short-term experimental diabetic retinopathy. Penn State Retina Research Group. Diabetes 47:815-820

24. Mizutani M, Gerhardinger C, Lorenzi M (1998) Muller cell changes in human diabetic retinopathy. Diabetes 47:445-449

25. Rungger-Brandle E, Dosso AA, Leuenberger PM (2000) Glial reactivity, an early feature of diabetic retinopathy. Invest Ophthalmol Vis Sci 41:1971-1980

26. Reichenbach A, Wurm A, Pannicke T, Iandiev I, Wiedemann P, Bringmann A (2007) Muller cells as players in retinal degeneration and edema. Graefes Arch Clin Exp Ophthalmol 245:627-636

27. Pannicke T, Iandiev I, Wurm A et al (2006) Diabetes alters osmotic swelling characteristics and membrane conductance of glial cells in rat retina. Diabetes 55:633-639

28. Ishii M, Fujita A, Iwai K et al (2003) Differential expression and distribution of Kir5.1 and Kir4.1 inwardly rectifying $\mathrm{K}^{+}$channels in retina. Am J Physiol Cell Physiol 285:C260-267

29. Kofuji P, Ceelen P, Zahs KR, Surbeck LW, Lester HA, Newman EA (2000) Genetic inactivation of an inwardly rectifying potassium channel (Kir4.1 subunit) in mice: phenotypic impact in retina. J Neurosci 20:5733-5740

30. Curtis TM, Hamilton R, Yong PH et al (2011) Muller glial dysfunction during diabetic retinopathy in rats is linked to accumulation of advanced glycation end-products and advanced lipoxidation endproducts. Diabetologia 54:690-698

31. Zhang Y, Xu G, Ling Q, Da C (2011) Expression of aquaporin 4 and Kir4.1 in diabetic rat retina: treatment with minocycline. J Int Med Res 39:464-479

32. Stitt AW, Curtis TM, Chen M et al (2016) The progress in understanding and treatment of diabetic retinopathy. Prog Retin Eye Res $51: 156-186$

33. Krady JK, Basu A, Allen CM et al (2005) Minocycline reduces proinflammatory cytokine expression, microglial activation, and caspase- 3 activation in a rodent model of diabetic retinopathy. Diabetes 54:1559-1565

34. Tang J, Kern TS (2011) Inflammation in diabetic retinopathy. Prog Retin Eye Res 30:343-358

35. Cheung AK, Fung MK, Lo AC et al (2005) Aldose reductase deficiency prevents diabetes-induced blood-retinal barrier breakdown, apoptosis, and glial reactivation in the retina of $\mathrm{db} / \mathrm{db}$ mice. Diabetes 54:3119-3125

36. Ghattas A, Lip PL, Lip GY (2011) Renin-angiotensin blockade in diabetic retinopathy. Int J Clin Pract 65:113-116

37. Cohn JN (2010) Role of the renin-angiotensin system in cardiovascular disease. Cardiovasc Drugs Ther 24:341-344

38. Wilkinson-Berka JL (2006) Angiotensin and diabetic retinopathy. Int J Biochem Cell Biol 38:752-765

39. Yacoub R, Campbell KN (2015) Inhibition of RAS in diabetic nephropathy. Int J Nephrol Renovasc Dis 8:29-40

40. Gilbert RE, Kelly DJ, Cox AJ et al (2000) Angiotensin converting enzyme inhibition reduces retinal overexpression of vascular endothelial growth factor and hyperpermeability in experimental diabetes. Diabetologia 43:1360-1367

41. Bui BV, Armitage JA, Tolcos M, Cooper ME, Vingrys AJ (2003) ACE inhibition salvages the visual loss caused by diabetes. Diabetologia 46:401-408

42. Hsu CR, Chen YT, Sheu WH (2015) Glycemic variability and diabetes retinopathy: a missing link. J Diabetes Complications 29: 302-306

43. Aiello LP, Ayala AR, Antoszyk AN et al (2015) Assessing the effect of personalized diabetes risk assessments during ophthalmologic visits on glycemic control: a randomized clinical trial. JAMA Ophthalmol 133:888-896 